August 1-5, 2021

\title{
Flow Field Study of a Top Heated Immiscible Liquid Layer Adjacent to Ice
}

\author{
Hamed F. Farahani ${ }^{* a}$, Tatsunori Hayashi ${ }^{\text {b }}$, Hirotaka Sakaue ${ }^{\text {b }}$, Ali S. Rangwala ${ }^{a}$ \\ a Department of Fire Protection Engineering, Worcester Polytechnic Institute, Worcester, MA \\ 01609, USA \\ a Department of Aerospace and Mechanical Engineering, University of Notre Dame, Notre \\ Dame, IN 46556, USA \\ *hfarmahinifaraha@wpi.edu
}

\begin{abstract}
A series of experiments were conducted to investigate the flow field of a top-heated liquid fuel adjacent to an ice block. The experimental setup consisted of a borosilicate container containing an ice wall and a layer of $n$-heptane heated from above. Particle Image Velocimetry (PIV) and Background Oriented Schlieren (BOS) measurements were conducted on the liquid-phase. PIV measurements showed a surface flow toward the ice caused by surface-tension forces, which is driven by the horizontal temperature gradients on the liquid surface. A recirculation zone was observed under the free surface and near the ice. The combination of the two flow patterns caused lateral intrusion in the ice, instead of a uniform melting across ice surface. BOS measurements indicated presence of density gradients below the free surface of $n$-heptane and in regions near the ice block. These density gradients were created by local small-scale temperature gradients. The current experiments were conducted to explore the processes that influence the ice melting by immiscible liquid layers.
\end{abstract}

\section{Introduction}

Arctic multi-year sea ice is already at risk of being lost due to global warming (Fig. 1). Spill of the oil in ice-infested waters of the Arctic is catastrophic for the environment. Oil spills contaminate the ocean and disturb ecosystems. Also, they bring about thermophysical alterations to the Arctic melting processes (Blankenet al., 2017). In particular, presence of oil adjacent to ice can fundamentally alter melting of ice sheets by increase of energy absorption from the sunlight. Also, spilled oil transfers this energy to the ice differently than the water. This is because, most hydrocarbon fuels are immiscible in water, thereby, melting of ice adjacent to immiscible liquids is dissimilar to conventional melting as the melt layer does not mix in the ambient liquid, i.e. without diffusion of melt. Additionally, interfacial forces between the two liquids and air can cause significant convective flows, which intensifies heat transfer to the ice (Farahani et al., 2017). Thus, understanding the heat transfer from an immiscible liquid to ice requires knowledge of the ambient 
$14^{\text {th }}$ International Symposium on Particle Image Velocimetry - ISPIV 2021

August 1-5, 2021

liquid flow field. The goal of the current study is to analyze the flow field of a liquid fuel that is adjacent to an ice wall and exposed to radiation from above, which mimic an oil spill scenario.

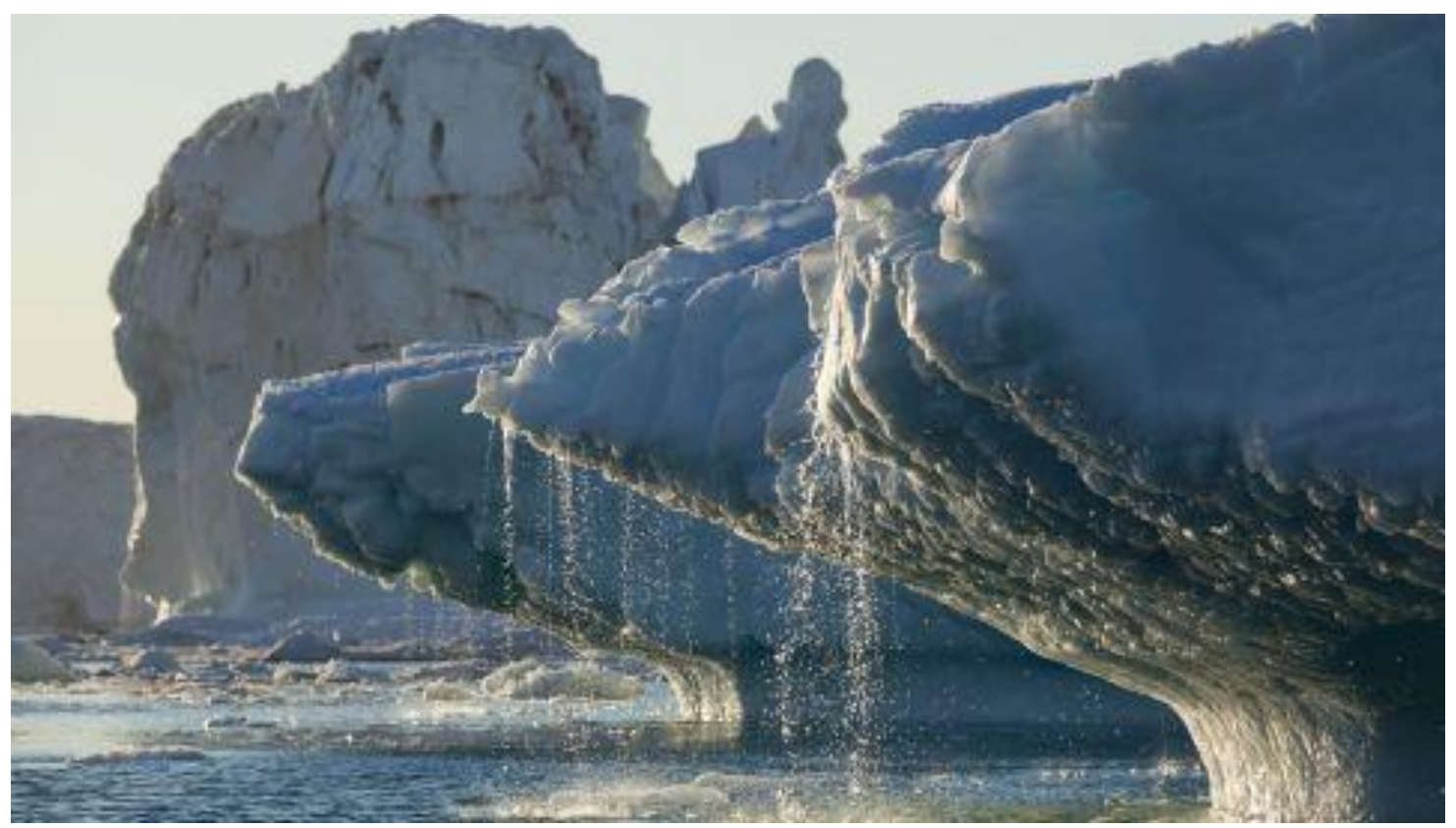

Figure 1: Melting of towering ice in Arctic in warm seasons (Baker, 2021).

\section{Experimental Setup}

Particle image velocimetry (PIV) and background oriented schlieren (BOS) measurement techniques were employed to study the flow field of a top-heated hydrocarbon liquid adjacent to an ice wall. Experiments were conducted under an IR radiant panel heater capable for delivery of a relatively uniform heat flux in the range of $1-30 \mathrm{~kW} / \mathrm{m}^{2}$. Below the IR heater panel, a squareshaped open top borosilicate container with side length of $10 \mathrm{~cm}$ and depth of $5 \mathrm{~cm}$ was used to hold the ice wall and the fuel as shown in Fig. 2. Experiment used a $10 \mathrm{~cm} \times 6 \mathrm{~cm} \times 3 \mathrm{~cm}$ ice wall placed on the left side of the container adjacent to $\sim 4 \mathrm{~cm}$ deep layer of n-heptane $\left(\mathrm{C}_{7} \mathrm{H}_{16}\right)$. The velocity field on the mid-plane of the liquid fuel perpendicular to the ice wall was obtained by PIV measurements. A laser sheet was produced by a green diode laser and seeding particles (glass spheres $10 \mu \mathrm{m}$ diameter) were added to the fuel layer prior to pouring it in the glass container. A DSLR camera with recording rate of $30 \mathrm{fps}$ was focused to the laser sheet. BOS experiments utilized the same camera and a randomly generated dotted camera as shown in Fig. 2 b-c. The BOS method attains the first spatial derivative of the index of refraction. The index of refraction is then related to density by the Gladstone-Dale relation. The video files were processed to obtained individual frames in black and white and the PIV post-process was performed by PIVLab (Thielicke and Stumhuis, 2014) on the obtained images. Cross correlation scheme with interrogation window size of 16 by 16 pixels was used for most of the postprocess efforts. 
August 1-5, 2021

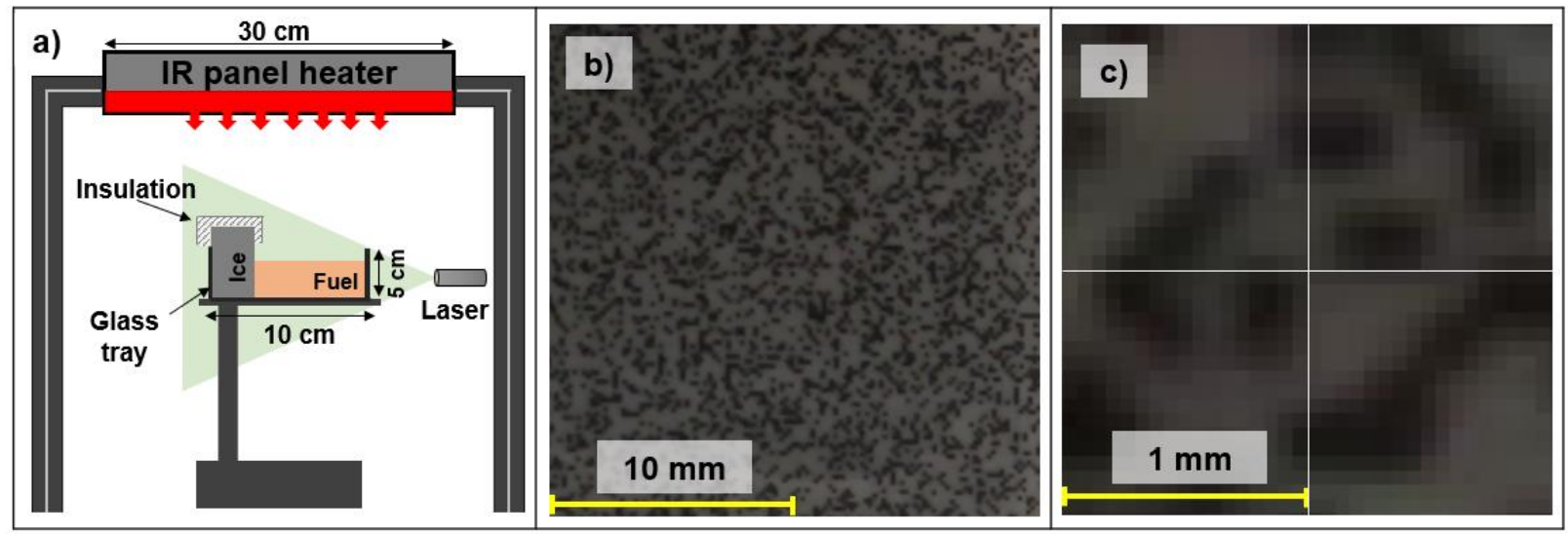

Figure 2: a) Schematics of the experimental apparatus (not to scale). b) Background of the random dot pattern used as imaged by the $1924 \times 1080$ pixels camera. The magnification(c) represents $32 \times 32$ pixels, and 4 interrogation windows of size $16 \times 16$ pixels.

\section{Results}

The radiation of the IR heater on the surface of n-heptane increased its temperature. This heat was transferred to the ice and provided the energy for the ice wall to start melting (Farahani et al., 2019). Figure 3 a-d shows side view of the ice wall and the sequence of its melting captured by the camera with intervals of 1 minute. Note that the ice wall is placed on the left and the liquid to a depth of $4 \mathrm{~cm}$ is on the right side separated with a solid yellow line. As can be seen in this figure, most of the melting occurred near the free surface of the liquid creating a lateral intrusion in the ice at the final stage of the melting (Fig. 3d). This means the heat transfer is mostly occurring near the free surface of the ice.
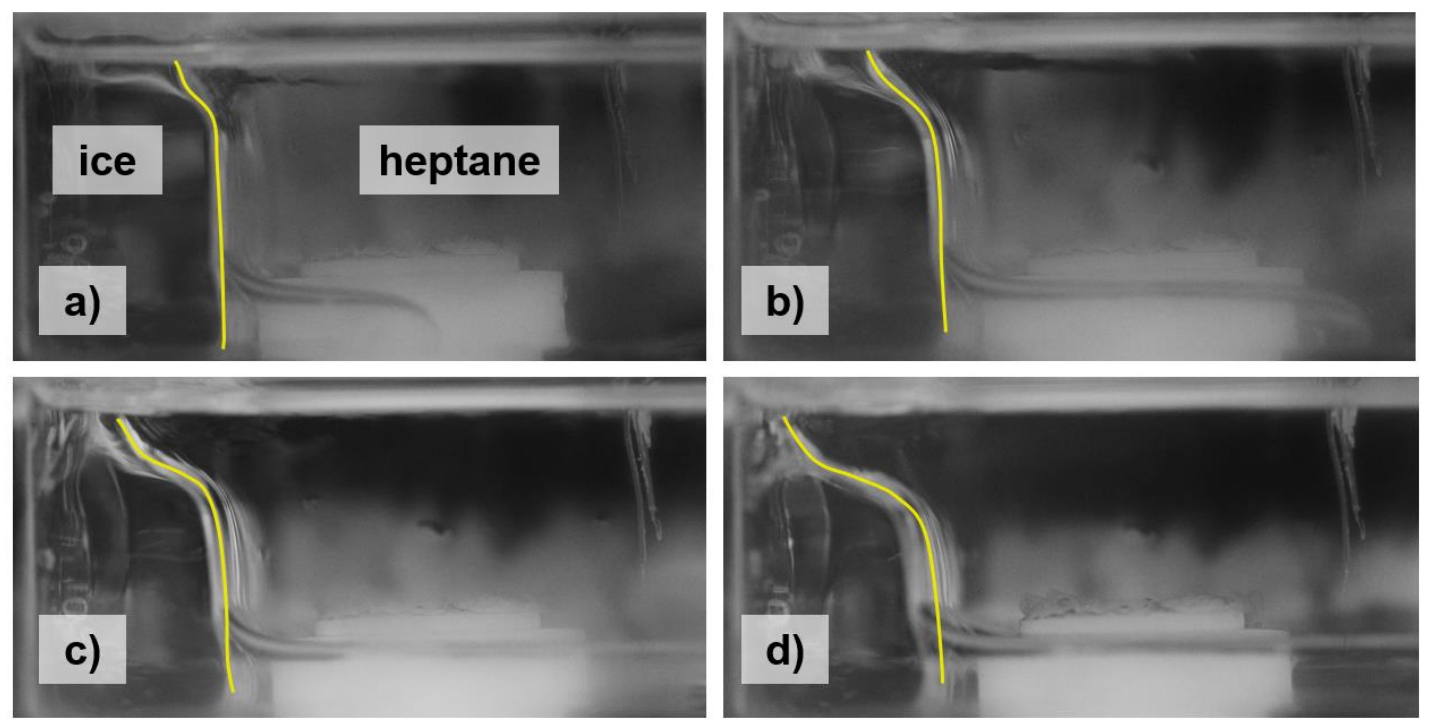

Figure 3: Sequence of melting shape of ice (left) adjacent to heptane (right) with approximately 1 -minute intervals under $3.1 \mathrm{~kW} / \mathrm{m}^{2}$ incident heat flux. 
14 ${ }^{\text {th }}$ International Symposium on Particle Image Velocimetry - ISPIV 2021

August 1-5, 2021

A previous parametric study on melting by immiscible layers has revealed that viscosity of liquid has a reverse correlation with melting rate (Farahani et al., 2018). The adverse correlation of the melting velocity to the viscosity implies that transfer of energy is mostly through convection rather conduction. PIV results of experiments with $n$-heptane exposed to radiation intensity of 1.5 and $3.1 \mathrm{~kW} / \mathrm{m}^{2}$ are shown in Fig. 4 and 5 . The ice is masked with color red. For the case of 1.5 $\mathrm{kW} / \mathrm{m}^{2}$, convective flows were mostly observed near the free surface of the liquid traveling toward the ice. This is due to the strong surface-tension forces at the interface of liquid-air towards the ice wall. The arriving flow stagnated near the ice and returned to the bulk of liquid forming a recirculation zone near the ice wall. Streamlines of vorticity show a strong recirculation zone at the free surface of the liquid and near the ice, as can be seen in Fig 4b. The velocity magnitude and vorticity in other regions of the liquid-phase were found to show minimal impact from the impinging radiation.

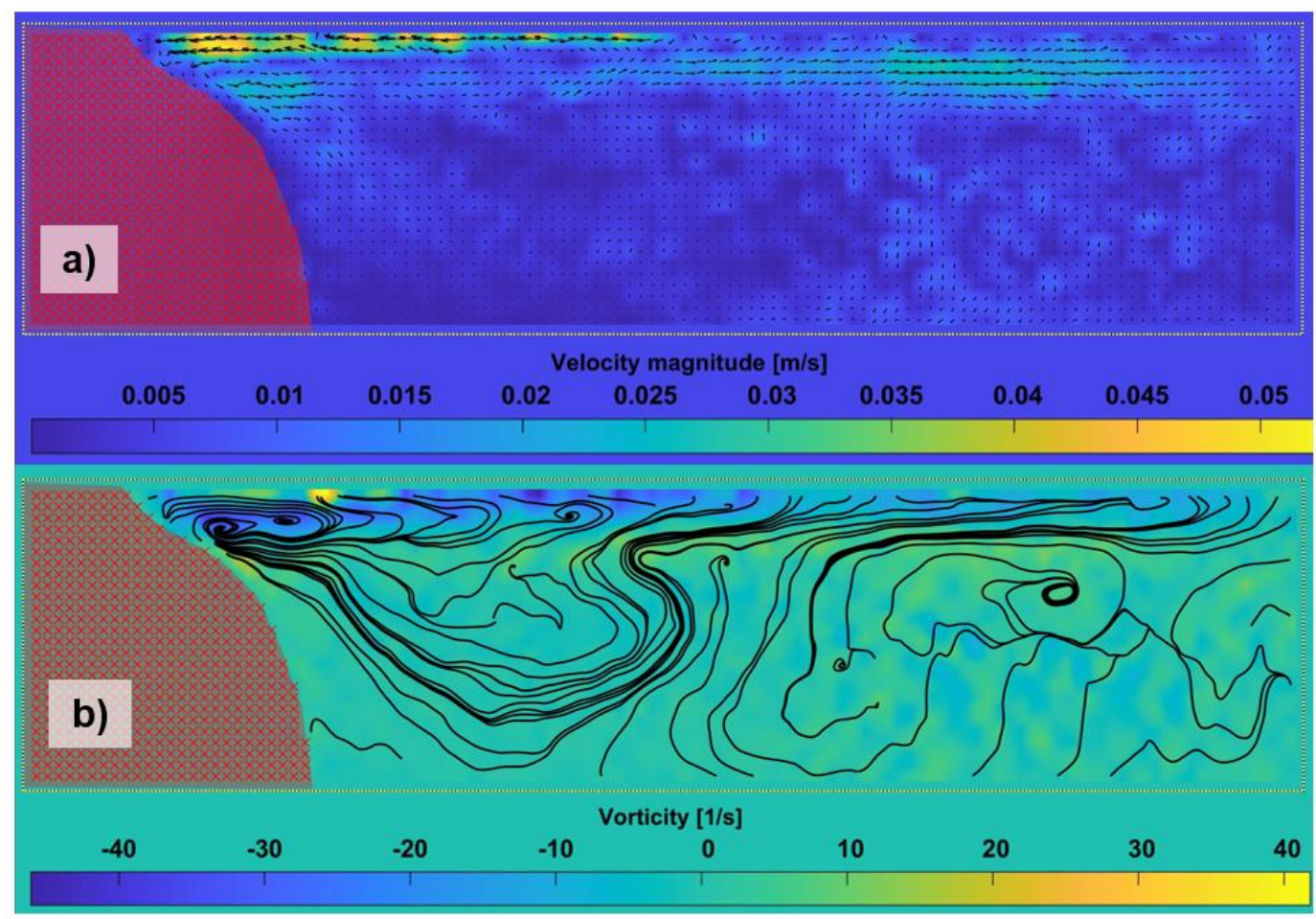

Figure 4: Flow field of $\mathrm{n}$-heptane exposed to $1.5 \mathrm{~kW} / \mathrm{m}^{2}$ radiation from above showing (a) vector field with background color map of velocity and (b) streamlines of vorticity with background of vorticity $(1 / \mathrm{s})$.

With increase of the radiation intensity, the melting rates were also increased. Figure 5a shows the vector field of $\mathrm{n}$-heptane exposed to $3.1 \mathrm{~kW} / \mathrm{m}^{2}$ radiation adjacent to an ice wall with background of velocity magnitude. Vector field of Figure 5a was averaged for 3 seconds due to optical distortions that eliminated a number of considerable number of particles from the field of view. As the heating intensity was increased, the density gradients that were created in the liquid caused optical interferences that adversely affects the PIV images. These interferences caused blockage and distortion of scattered lights from seeding particles. Thus, portion of the signal was 
14 ${ }^{\text {th }}$ International Symposium on Particle Image Velocimetry - ISPIV 2021

August 1-5, 2021

lost and obtaining the full image of the vector field was not possible. Averaging in this situation provided an opportunity to see the general flow field, however, velocity magnitudes reported for the average results are reduced by averaging. Figure $5 \mathrm{~b}$ shows the streamlines of vorticity in a background of vorticity map. As can be seen, the recirculation zone near the ice is present and the downward directed flow created another recirculation zone deeper in the liquid.

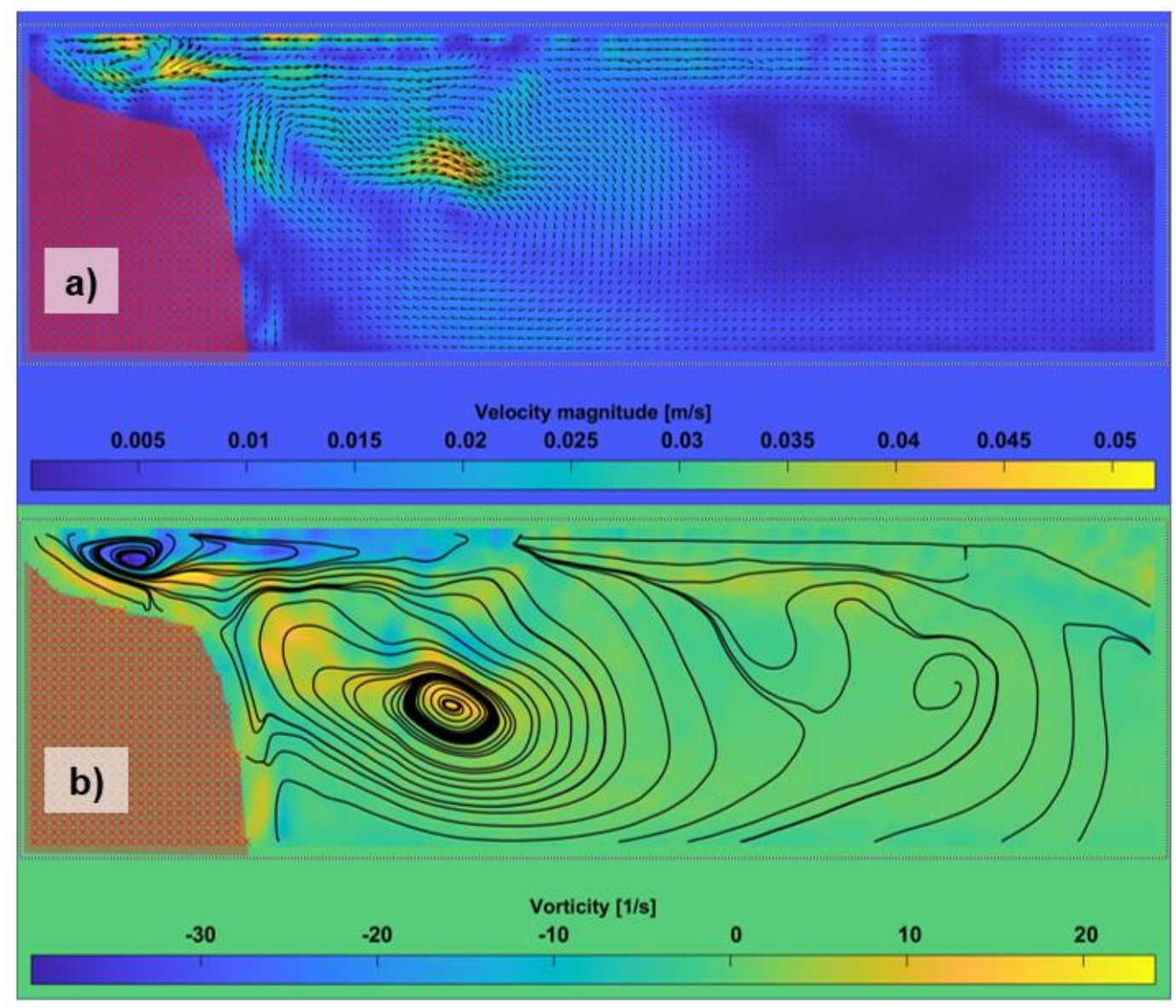

Figure 5: Flow field of $\mathrm{n}$-heptane exposed to $3.1 \mathrm{~kW} / \mathrm{m}^{2}$ radiation from above showing (a) vector field with background color map of velocity and (b) streamlines of vorticity with background of vorticity $(1 / \mathrm{s})$.

Assessment of the PIV results show that regions of higher melting rate correspond to locations of higher liquid velocity and vorticity. However, due to presence of density gradients, the optical field is distorted and thereby scattered light from seeding particles are not captured entirely. This effect is intensified at higher radiation levels, which renders PIV measurement unusable. Nevertheless, averaging the results gives a qualitative understanding of flow patterns.

Loss of PIV signal, which has also been observed previously in a similar type experiments [4], is due to significant temperature differences that are caused by impingements of the IR heater and presence of ice. The intrinsic thermal disparity of this system leads to density gradients and subsequently change in the index of refraction of the liquid that causes distortion and obstructions 
14 ${ }^{\text {th }}$ International Symposium on Particle Image Velocimetry - ISPIV 2021

August 1-5, 2021

in the field of view. Although it is not possible to correct these issues occurring for PIV, BOS can be used to identify regions of significant gradients. As can be seen in Fig. 6 from the results of BOS measurements (reported by pixel/frame displacement), density gradients are mostly present below the surface of the liquid and near the ice wall, which exactly overlaps with the regions of weak PIV signal.

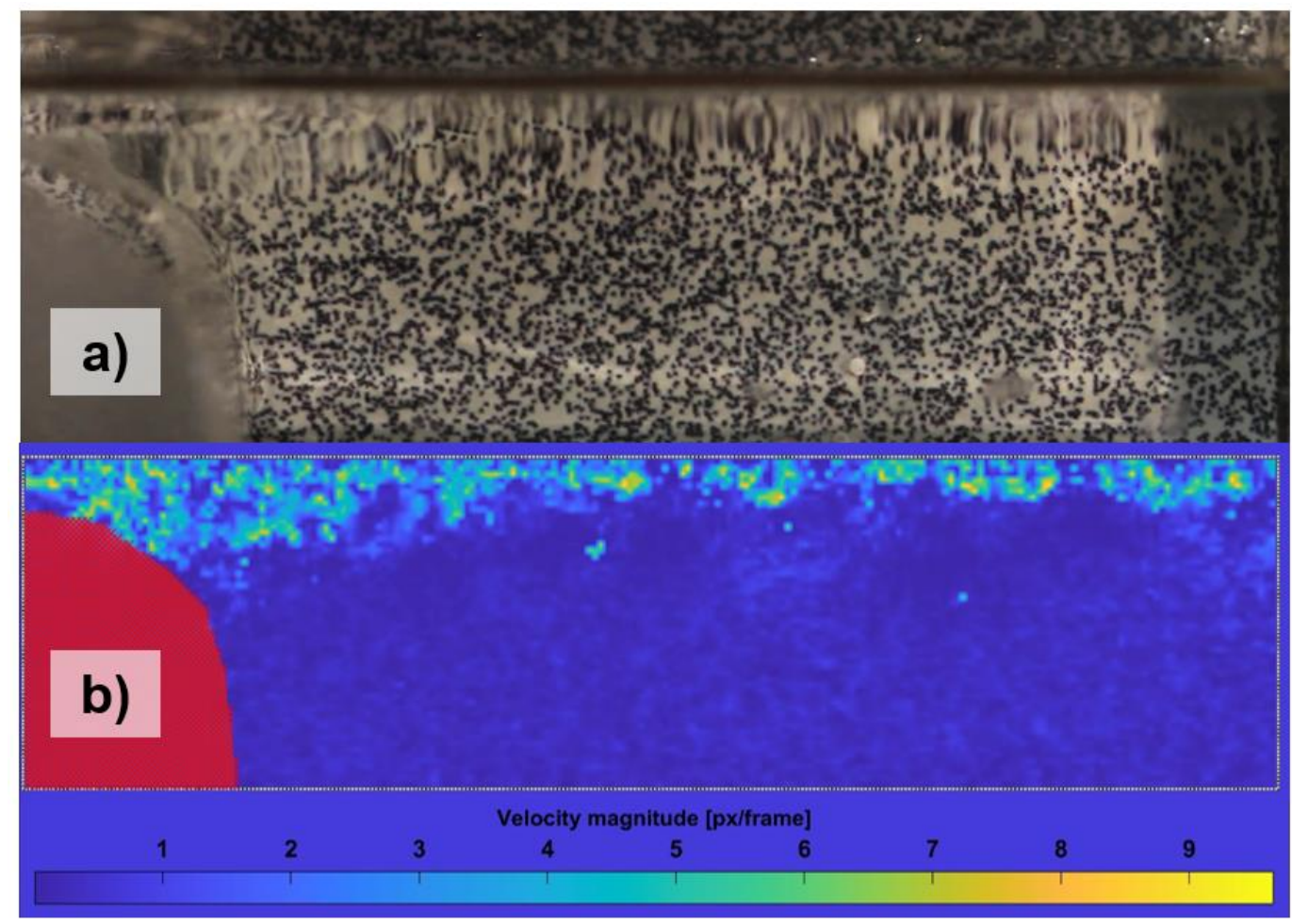

Figure 6. a) Raw background image of BOS showing distortions of the pattern near free surface, b) instantaneous, color map of pixel displacement (pixel/frame) in n-heptane.

\section{Conclusions}

A series of experiments were conducted to investigate the flow field of an immiscible liquid layer exposed to radiation from above. Fluid flow characteristics of the adjacent immiscible nheptane layer were obtained by using PIV and BOS methods. PIV measurements of convective flows in the immiscible liquid showed a surface flow toward the ice, which is caused by surfacetension forces. Presence of recirculation zones enhanced the melting and assisted the return flow in the bulk of the liquid. The results indicated the combination of PIV and BOS techniques is an effective method in scrutinizing the liquid-phase convection and identifying the regions with temperature gradients. However, acquiring quantitative data can be difficult due the density gradients that cause significant distortions in the field of view. The qualitative results that are obtained allow for an informed analysis of heat transfer from the liquid to the ice. Understanding the melting heat transfer by immiscible liquids is a necessary step toward understanding the 
$14^{\text {th }}$ International Symposium on Particle Image Velocimetry - ISPIV 2021

August 1-5, 2021

impacts of oil spills in the Arctic. This research is planned to continue with the mentioned experiments to obtain more results with the combination of the two techniques.

\section{Acknowledgement}

This material is based upon work supported by the National Science Foundation under Grant No. 1938980. Any opinions, findings and conclusions or recommendations expressed in this material are those of the author(s) and do not necessarily reflect those of the National Science Foundation.

\section{References}

Blanken, H., et al., Modelling the long-term evolution of worst-case Arctic oil spills. Marine pollution bulletin, 2017. 116(1-2): p. 315-331.

Baker, H., https://www.livescience.com/amp/arctic-ice-arches-melting-fast.html

Yamada, M., et al., (1998) Melting heat transfer characteristics of a horizontal ice cylinder immersed in an immiscible liquid. 27(5): p. 336-352.

Farahani, H.F. Fu, Y., Jomaas, G., Rangwala A., (2018) Convection-driven cavity formation in ice adjacent to externally heated flammable and non-flammable liquids. Cold Regions Science and Technology, 2018. 154: p. 54-62.

Farahani, H.F., Alva, W., Rangwala, A., Jomaas G., (2017), Convection-driven melting in an noctane pool fire bounded by an ice wall. Combustion and Flame, 179: p. 219-227.

Farahani, H. F., Torero, J. L., Jomaas, G., Rangwala, A., (2019) Scaling analysis of ice melting during burning of oil in ice-infested waters, International Journal of Heat and Mass Transfer, Volume 130, Pages 386-392.

Thielicke, W., and Stamhuis E. J., (2014) "PIVlab - Towards User-friendly, Affordable and Accurate Digital Particle Image Velocimetry in MATLAB," Journal of Open Research Software, vol. 2. 